\title{
MODIFIED NONLINEAR SCHRÖDINGER EQUATION FOR NONLINEAR WAVES IN OPTICAL FIBRES
}

\author{
K. MURaWsKi \\ Department of Mathematical Sciences, University of St Andrews \\ St Andrews, KY16 9SS, Scotland \\ (Received August 30, 1989; in revised version April 16, 1991)
}

\begin{abstract}
A new model equation governing the propagation of nonlinear pulses in optical fibres has been derived on the assumption of a saturated nonlinearity of the refractive index. This equation is a combination of the exponential nonlinear Schrödinger equation and the derivative one. It is valid for the long fibres. A modulational stability has been calculated to find out a cut-off in an angular frequency of a carrier wave. Moreover, it has been shown that the equation possesses family of stationary solutions. An initial value problem has been discussed on the basis of the implicit pseudo-spectral scheme.
\end{abstract}

PACS numbers: 03.40.Kf, 42.65.-k, 52.35.Mw

\section{Introduction}

Nonlinear waves in optical fibres have been the subject of a number of experimental [1-7] and theoretical studies [8-13]. Owing to the Kerr effect, the refractive index of the fibre quadratically depends on the electric field amplitude of the optical pulses. In a weakly nonlinear, dispersive medium, the pulse propagation is governed by the cubic nonlinear Schrödinger (cNS) equation $[14,15]$. When the effects of dispersion and nonlinearity are exactly balanced, localized optical pulses can propagate in the form of solitons.

The cNS equation is valid for short samples. An extension of the theory to long fibres leads to a model equation which is a combination of the cNS equation and the derivative one [16]. These equations are derived, however, assuming the quadratic dependence of the index of refraction on intensity of the light and thus are valid for small amplitude waves. In this paper we extend this theory deriving an equation which leads to the cNS equation in the limit of small amplitude waves. 
The paper is organized as follows. The next section presents the derivation of the model equation assuming exponential dependence of the refractive index on the electric field. Section 3 shows a necessary condition for the modulational stability of plane waves as solutions of this equation. The existence of stationary wave solutions is proved in Section 4. A pseudo-spectral method (e.g. [17]) is applied in Section 5 to study an initial-value problem. The final part consists of a short summary.

\section{Derivation of modified nonlinear Schrödinger equation}

We consider one-dimensional wave propagation in a monomode dielectric waveguide. The linearly polarized electric field $E(x, t)$ is described then by the scalar equation

$$
E_{x x}-\frac{1}{c^{2}} D_{\mathrm{L}_{t t}}=\frac{2 n_{0} n_{2}}{c^{2}}\left[\left(1-\mathrm{e}^{-|E|^{2}}\right) E\right]_{t t},
$$

which follows from the Maxwell equations. Subscripts $x$ and $t$ indicate the partial derivatives. $c$ and $D_{\mathrm{L}}$ are the light velocity and the linear displacement, respectively. The Kerr coefficient $n_{2}$ represents the nonlinear part of the refractive index $n[18]$,

$$
n=n_{l}+n_{2}\left(1-\mathrm{e}^{-|E|^{2}}\right) .
$$

$n_{l}$ is a linear part of the refractive index and

$$
n_{0}=n_{l}\left(\omega_{0}\right)
$$

where $\omega_{0}$ is a frequency of the electric field.

In the above formulae, for the sake of simplicity, we have neglected dielectric losses which, in the linear regime of mono-mode fibres, are typically in the range $0.2-1.0 \mathrm{~dB} / \mathrm{km}$. Furthermore, we have assumed a saturated nonlinearity instead of the commonly applied quadratic Kerr effect. To prove that our assumption is more general than usually used one we rewrite the formula (2.2) in the small amplitude limit. We find that the refractive index $n$ quadratically depends on the electric field $E$,

$$
n=n_{l}+2 n_{2}|E|^{2} .
$$

The saturated nonlinearity has been already applied in a nonlinear optics context by Murawski and Koper [18] and in plasma physics by D'Evelyn and Morales [19] and Kaw et al. [20]. We mention as well that in the fundamental experiment of Mollenauer et al. [1] $n_{0}$ and $n_{2}$ have been chosen as follows $n_{0}=1.45$ and $2 n_{2}=1.1 \times 10^{-13}$.

Additionally, we make an assumption that

$$
\omega_{\mathrm{i}} \ll \omega \ll \omega_{\mathrm{e}},
$$

where $\omega$ is the operating frequency, $\omega_{\mathrm{i}}$ and $\omega_{\mathrm{e}}$ are the ionic and electronic frequencies, respectively. This is very good approximation for $\mathrm{SiO}_{2}$ glasses and implies that the dielectric is weakly dispersive. 
To continue our search for a model equation, we write the electric field in the form

$$
E(x, t)=u(x, t) \mathrm{e}^{\mathrm{i}\left(q x-\omega_{0} t\right)},
$$

where $q$ is the propagation constant. Now, we are able to define a linear part of the displacement vector $D_{\mathrm{L}}$,

$$
D_{\mathrm{L}}=\int_{-\infty}^{t} n_{l}^{2}\left(t-t^{\prime}\right) E\left(t^{\prime}\right) \mathrm{d} t^{\prime}
$$

Substitution of Eqs. (2.5) and (2.6) into Eq. (2.1) leads to the equation for a slowly varying envelope $u(x, t)$

$$
\begin{aligned}
& {\left[\partial_{x}^{2}+2 \mathrm{i} q \partial_{x}-q^{2}+k_{0}{ }^{2}+2 \mathrm{i} k_{0} k_{0}{ }^{\prime} \partial_{t}-\left(k_{0}^{\prime 2}+k_{0} k_{0}^{\prime \prime}\right) \partial_{t}{ }^{2}\right] u} \\
& =\frac{2 n_{0} n_{2}}{c^{2}} \mathrm{e}^{\mathrm{i} \omega_{0} t}\left[\left(1-\mathrm{e}^{-|u|^{2}}\right) u \mathrm{e}^{-\mathrm{i} \omega_{0} t}\right]_{t t},
\end{aligned}
$$

where

$$
k^{2}=\frac{\omega^{2} n_{l}^{2}}{c^{2}}, \quad k_{0}^{2}=\frac{\omega_{0}^{2} n_{0}^{2}}{c^{2}}, \quad k_{0}^{\prime}=\left.\frac{\partial k}{\partial \omega}\right|_{\omega_{0}}, \quad k_{0}^{\prime \prime}=\left.\frac{\partial^{2} k}{\partial \omega^{2}}\right|_{\omega_{0}}
$$

and $\partial$ means the partial derivatives operator. For long enough samples the right hand side of (2.7) may be replaced by

$$
-2 \frac{n_{2}}{n_{0}} k_{0}{ }^{2}\left(1-\mathrm{e}^{-|u|^{2}}\right) u-4 \mathrm{i} \frac{n_{2}}{c} k_{0}\left[\left(1-\mathrm{e}^{-|u|^{2}}\right) u\right]_{t} \text {. }
$$

To simplify further Eq. (2.7) we also use the slowly varying envelope approximation

$$
k_{0} \gg \partial_{x}-2 k_{0}^{\prime} \partial_{t} \text {. }
$$

It implies that the changes of $u(x, t)$ per wavelength are extremely small, which is in full agreement with the experimental observations.

Finally, we obtain from (2.7)

$$
\mathrm{i}\left(u_{x}+k_{0}^{\prime} u_{t}\right)-\frac{1}{2} k_{0}^{\prime \prime} u_{t t}+\frac{\omega_{0} n_{2}}{c}\left(1-\mathrm{e}^{-|u|^{2}}\right) u+2 \mathrm{i} \frac{n_{2}}{c}\left[\left(1-\mathrm{e}^{-|u|^{2}}\right) u\right]_{t}=0 .
$$

Rewriting this equation in coordinates of the moving frame,

$$
\tau=t-k_{0}^{\prime} x, \quad x=x,
$$

we arrive at the model equation,

$$
\mathrm{i} u_{x}+\alpha u_{\tau \tau}+\beta\left(1-\mathrm{e}^{-|u|^{2}}\right) u+\mathrm{i} \gamma\left[\left(1-\mathrm{e}^{-|u|^{2}}\right) u\right]_{\tau}=0,
$$

where:

$$
\alpha=-\frac{1}{2} k_{0}^{\prime \prime}, \quad \beta=\frac{\omega_{0} n_{2}}{c}, \quad \gamma=2 \frac{n_{2}}{c} .
$$

For small amplitudes $u(x, \tau), 1-\mathrm{e}^{-|u|^{2}}$ reduces to $|u|^{2}$ and we have equation derived e. g. by Shukla and Rasmussen [16] and Jain and Tzoar [14]. For short enough samples the last term of Eq. (2.11) may be neglected and we get the exponential nonlinear Schrödinger equation studied recently by Murawski and Koper [18]. 


\section{Modulational instabilities}

Modulational instability has been studied in many areas of physics [21-23]. The effect of time derivative nonlinearity on the modulational instability of coherent signals in optical fibres has been investigated by Shukla and Rasmussen [16]. The modulational instability in lossy fibres has been discussed by Anderson and Lisak [15]. It relays on a process in which small amplitude perturbations from the steady-state grow exponentially as a result of an interaction between Fourier modes.

Equation (2.11) has a steady-state ( plane-wave) solution corresponding to the wave of a constant amplitude:

$$
u=A \mathrm{e}^{\mathrm{i} a x},
$$

where $A$ is an arbitrary constant and

$$
a=\beta\left(1-\mathrm{e}^{-A^{2}}\right) .
$$

We perturb the plane wave, given by (3.1), by the small amplitude disturbance $v(x, \tau)$

$$
u(x, \tau)=(A+v) \mathrm{e}^{\mathrm{i} a x} .
$$

Substitution of this equation into Eq. (2.11) leads to the linearized equation for $v(x, \tau)$ :

$$
\begin{aligned}
& \mathrm{i} v_{x}-a v+\alpha v_{\tau \tau}+\beta \mathrm{e}^{-A^{2}} A^{2}\left(v+v^{*}\right)+\beta\left(1-\mathrm{e}^{-A^{2}}\right) v \\
& +\mathrm{i} \gamma \mathrm{e}^{-A^{2}}\left[\left(A^{2}-1\right) v_{\tau}+A^{2} v^{*}{ }_{\tau}\right]+\mathrm{i} \gamma v_{\tau}=0 .
\end{aligned}
$$

Furthermore, we introduce the real and imaginary parts of $v$ by means of $v(x, \tau)=$ $R(x, \tau)+\mathrm{i} I(x, \tau)$ and look for solutions of the form

$$
R, I \sim \mathrm{e}^{\mathrm{i}(k x-\omega \tau)},
$$

where $k$ and $\omega$ represent the wave number and the frequency of the modulation, respectively.

Substituting Eq. (3.5) into Eq. (3.4), we get the dispersion relation

$$
\begin{aligned}
& k^{2}-2 \gamma \omega\left[1-\left(1-A^{2}\right) \mathrm{e}^{-A^{2}}\right]-\alpha^{2} \omega^{4} \\
& +\omega^{2}\left[2 \alpha \beta A^{2} \mathrm{e}^{-A^{2}}+\gamma^{2}\left(1-\mathrm{e}^{-A^{2}}\right)^{2}+2 \gamma^{2} A^{2} \mathrm{e}^{-A^{2}}\left(1-\mathrm{e}^{-A^{2}}\right)\right]=0 .
\end{aligned}
$$

This equation admits of an oscillatory instability for

$$
\begin{aligned}
& \alpha^{2} \omega^{2} \leq 2 \alpha \beta A^{2} \mathrm{e}^{-A^{2}}+\gamma^{2}\left(1-\mathrm{e}^{-A^{2}}\right)^{2} \\
& +2 \gamma^{2} A^{2} \mathrm{e}^{-A^{2}}\left(1-\mathrm{e}^{-A^{2}}\right)-\gamma^{2}\left[1-\left(1-A^{2}\right) \mathrm{e}^{-A^{2}}\right]^{2} \equiv \alpha^{2} \omega_{\mathrm{c}}{ }^{2} .
\end{aligned}
$$

The corresponding expression for the exponential nonlinear Schrödinger equation without the derivative term is readily obtained by setting $\gamma=0$. See Murawski and Koper [18]. The effect of finite $\gamma$ is to reduce the region of modulational stability. 


\section{Stationary wave solutions}

We look for stationary wave solutions of Eq. (2.11) in the form

$$
u=u_{0}(\xi) \mathrm{e}^{\mathrm{i}(a x+\phi(\xi))},
$$

where the stretched variables,

$$
\xi=\tau+c x, \quad x=x,
$$

have been introduced. A value of $-1 / c$ is the velocity of a stationary wave and $a$ is an arbitrary constant.

Substituting Eq. (4.1) into Eq. (2.11), we get a couple of equations for the envelope $u_{0}(\xi)$ and the phase $\phi(\xi)$ :

$$
\begin{aligned}
& \left(c+2 \alpha \phi_{\xi}\right) u_{0 \xi}+\gamma\left[\left(1-\mathrm{e}^{-u_{0}^{2}}\right) u_{0}\right]_{\xi}+\alpha u_{0} \phi_{\xi \xi}=0, \\
& \alpha u_{0 \xi \xi}-\left(a+c \phi_{\xi}+\alpha \phi_{\xi}^{2}\right) u_{0}+\left(\beta+\gamma \phi_{\xi}\right)\left(1-\mathrm{e}^{-u_{0}^{2}}\right) u_{0}=0 .
\end{aligned}
$$

After a multiplication of Eq. (4.3) by $u_{0}$ and an integration of such obtained one we get

$$
2 \alpha u_{0}^{2} \phi_{\xi}=\gamma \mathrm{e}^{-u_{0}^{2}}+\left[\gamma\left(2 \mathrm{e}^{-u_{0}^{2}}-1\right)-c\right] u_{0}^{2}+A,
$$

where $A$ is an integration constant. Equation (4.4) leads then to the equation for $u_{0}$ :

$$
\begin{aligned}
& 2 \alpha^{2} u_{0 \xi \xi}=a_{1} u_{0}+\frac{A^{2}}{2 u_{0}^{3}}+a_{3} u_{0} \mathrm{e}^{-u_{0}^{2}}+\frac{A \gamma}{u_{0}} \mathrm{e}^{-u_{0}^{2}}\left(1+\frac{1}{u_{0}^{2}}\right) \\
& +\frac{\gamma^{2}}{u_{0}} \mathrm{e}^{-2 u_{0}{ }^{2}}\left(1+\frac{1}{2 u_{0}{ }^{2}}\right),
\end{aligned}
$$

where we introduced the following description:

$$
a_{1}=\frac{1}{2}\left[4 \alpha(a-\beta)-\gamma(\gamma+2 c)-c^{2}\right] ; \quad a_{2}=\gamma(\gamma+c)+2 \alpha \beta .
$$

Multiplying this equation by $2 u_{0 \xi}$ and integrating, we obtain

$$
2 \alpha^{2} u_{0 \xi}{ }^{2}=a_{1} u_{0}^{2}-\frac{A^{2}}{2 u_{0}^{2}}-\left(a_{2}+\frac{A \gamma}{u_{0}^{2}}\right) \mathrm{e}^{-u_{0}^{2}}-\gamma^{2} \frac{\mathrm{e}^{-2 u_{0}^{2}}}{2 u_{0}^{2}}+l \equiv Y\left(u_{0}\right),(4.7)
$$

where $l$ is another integration constant.

The qualitative nature of the solution of Eq. (2.11) may be determined from consideration of the function $Y\left(u_{0}\right)$ which should be bounded for bounded $u_{0}$ and must possess double roots. It happens when

$$
Y^{\prime}\left(u_{0}\right)=Y\left(u_{0}\right)=0 .
$$

The one and two double roots usually correspond to the soliton or shock wave. Other values of $l$ for which $Y\left(u_{0}\right)>0$ correspond to periodic waves. Thus suitably 
choosing values of the parameters, it is possible to find such solutions. Unfortunately, analytical forms of these solutions do not seem to exist. So, we simply present two families of solutions. We thus have a range of periodic waves which are limited by the linear wave, for $l=l_{\min }$, and the soliton for $l=l_{\max }$. $l_{\min }$ and $l_{\max }$ are minimum and maximum values of $l$ calculated from equations $Y\left(u_{0}\right)=Y^{\prime}\left(u_{0}\right)=0$. See Fig. 1 for the dependence of $Y$ on $u_{0}$. Another case is shown in Fig. 2. We have got here only periodic waves like solutions of Eq. (4.7). Note the singularity at the point $u_{0}=0$ in both cases. A case of $\gamma=0$ corre-
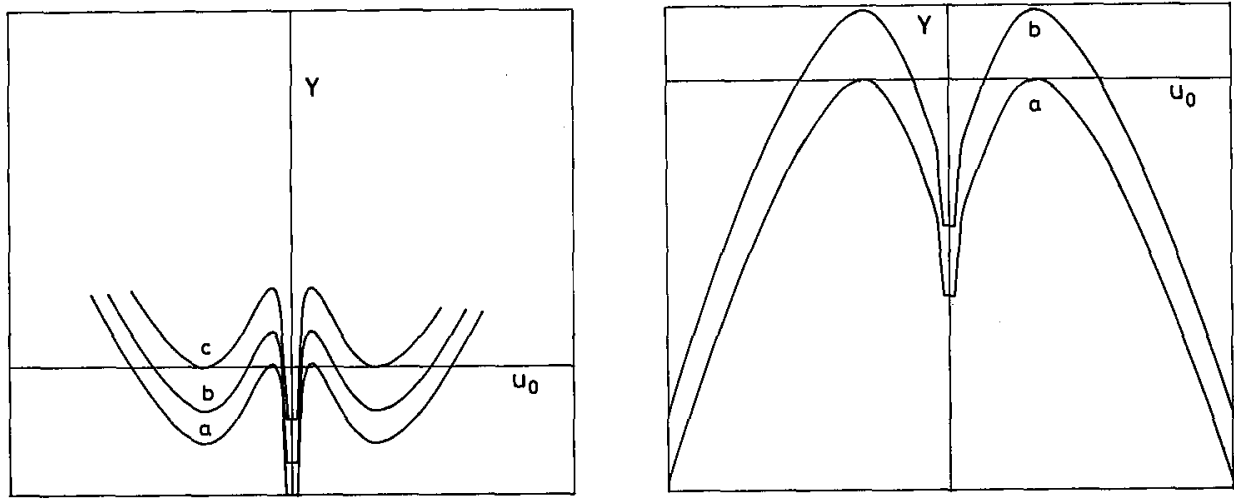

Fig. 1. Phase diagrams for the exponential derivative nonlinear Schrödinger equation $\left(a_{1}>0\right)$ : curve $a$, linear wave limit; curve $b$, cnoidal wave; curve c, soliton Fig. 2. As for Fig. 1, but $\left(a_{1}<0\right)$ : curve a, linear wave limit; curve b, cnoidal wave.

sponds to the exponential nonlinear Schrödinger equation whose stationary wave solutions have been discussed by Murawski and Koper [18].

\section{Numerical results}

The numerical code utilizes the implicit method in an integration with respect to $x$. The nonlinear terms are integrated in a configuration space transforming $u$ back and forth between real and Fourier space using fast Fourier transformations to calculate derivatives with respect to $\tau$. The number of mesh points in one period of $\tau$ has been to be chosen 64 .

Equation (2.11) is discretized as follows:

$$
\begin{aligned}
& \mathrm{i}\left(u_{j}{ }^{m+1}-u_{j}{ }^{m}\right) / \Delta x+\alpha F^{-1}(\mathrm{i} n)^{2} F{u^{\prime}}^{m}+\beta\left(1-\mathrm{e}^{-\left|u_{j}^{\prime m}\right|^{2}}\right) u_{j}^{\prime m} \\
& +\mathrm{i} \gamma F^{-1} \mathrm{i} n F\left(1-\mathrm{e}^{-\left|u_{j}^{\prime m}\right|^{2}}\right) u_{j}^{\prime m}=0,
\end{aligned}
$$

where

$$
u_{j}^{m}=u(m \Delta x, j \Delta \tau), \quad u_{j}^{\prime m}=\left(u_{j}^{m+1}+u_{j}^{m}\right) / 2 .
$$


$F$ and $F^{-1}$ are Fourier and inverse Fourier operators. By this way all quantities are centered around $u_{j}{ }^{m+\frac{1}{2}}$. For every space step, $\Delta x$, the transcendental Eq. (5.1) was solved by minimization procedure.

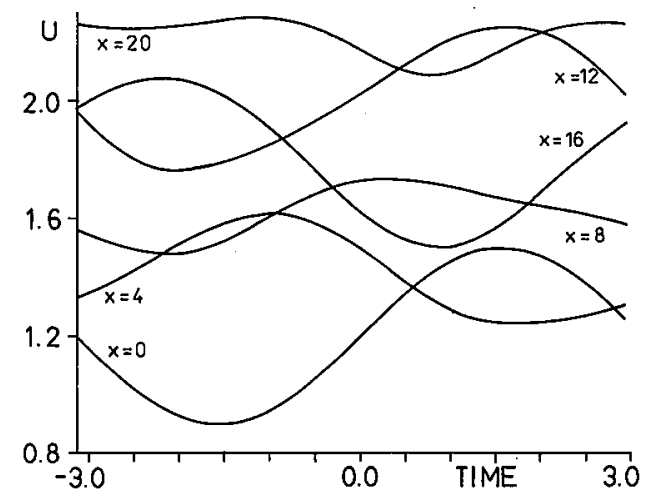

Fig. 3. Panoramic view of modulation envelope $|u|$ for $u(\tau, x=0)=1+0.3 \sin \tau$ and $\alpha=-1, \beta=\gamma=1$. Profiles of the envelope at $x=0,4,8,12,16$, and 20.

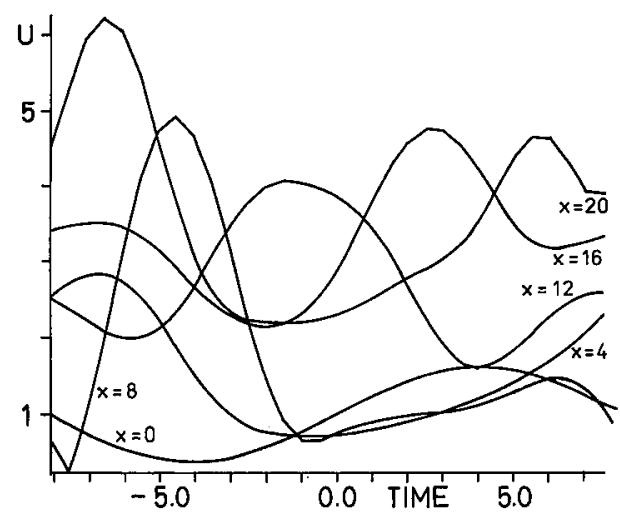

Fig. 4. Panoramic display of dynamical evolution of the sine-type disturbance $u(\tau, x=0)=1+0.3 \sin (\omega \tau)$, where $\omega=\omega_{c} / 2$ (see (3.7)). Profiles of modulation envelope at $x=0,4,8,12,16$, and 20. $\alpha=\beta=\gamma=1$.

We have performed standard numerical test doubling the number of Fourier modes and halving the integration step for $x$ until no significant changes has appeared. Numerically obtained results have been verified, however, by calculation of the integral

$$
\int_{-\infty}^{\infty}|u|^{2} \mathrm{~d} \tau
$$


as a conserved quantity. The error in the calculations has been less than $1 \%$.

Figures 3 and 4 show typical results of the computation. The sine-type initial disturbance deformes slightly at a longer distance of propagation. Only the phase changes and the recurrence period is about 12 . So, the disturbance is stable (Fig. 3 ). Unstable disturbance is presented in Fig. 4. It is clearly seen that dressed solitons are created from the sine-type initial condition at $x=8$ and $x=20$.

\section{Summary}

Based on the rigorous development of the nonlinear optics method, we have derived the model equation which describes the propagation of coherent optical pulses in the nonlinear fibres. This equation consists of a combination of the exponential nonlinear Schrödinger equation and the derivative one. It is valid for wide range of waves and particularly for long samples and large amplitudes of the incident electric field. Although this equation is also valid for short samples, it transforms to the exponential nonlinear Schrödinger equation. In the limit of small amplitude waves we may replace it by the cNS equation and the derivative one.

We have shown that the inclusion of the derivative term in the exponential derivative nonlinear Schrödinger equation influences modulational stability limiting its regime.

We have also proved an existence of stationary waves as solutions of this equation. Additionally, the pseudo-spectral method has been applied to solve an initial-value problem for the equation we derived.

\section{References}

[1] L.F. Mollenauer, R.H. Stolen, J.P. Gordon, Phys. Rev. Lett. 45, 1095 (1980).

[2] G.P. Agrawal, P.L. Baldeck, R.R. Alfano, Opt. Lett. 14, 137 (1989).

[3] L.F. Mollenauer, Optics News, May, 42 (1986).

[4] A. Hasegawa, Y. Kodama, Proc. IEEE 69, 1145 (1981).

[5] Y. Kodama, J. Stat. Phys. 39, 597 (1985).

[6] Y. Kodama, A. Hasegawa, IEEE J. Quantum Electronics QE-23, 510 (1987).

[7] C.R. Menyuk, IEEE J.Quantum Electronics QE-23, 174 (1987).

[8] G.P. Agrawal, Phys. Rev. Lett. 59, 880 (1987).

[9] S. Wabnitz, Phys. Rev. A 38, 2018 (1988).

[10] M.V. Tratnik, J.E. Sipe, Phys. Rev. A 38, 2011 (1588).

[11] A.L. Berkhoer, V.E. Zakharov, Sov. Phys. JETP 31, 486 (1970).

[12] Y. Inoue, J. Phys. Soc. Japan 43, 243 (1977).

[13] R.H. Enns, S.S. Rangnekar, A.E. Kaplan, Phys. Rev. A 36, 1270 (1987).

[14] M. Jain, N. Tzoar, J. Appl. Phys. 49, 4649 (1978).

[15] D. Anderson, M. Lisak, Opt. Lett. 9, 468 (1984). 
[16] P.K. Shukla, J.J. Rasmussen, Opt. Lett. 11, 171 (1986).

[17] B. Fornberg, G.B. Whitham, Philos. Trans. R. Soc. Lond. A 289, 373 (1978).

[18] K. Murawski, Z.A. Koper, Ann. Physik (1991), in press.

[19] M. D'Evelyn, G.J. Morales, Phys. Fluids 21, 1997 (1978).

[20] P. Kaw, G. Schmidt, T. Wilcox, Phys. Fluids 16, 1522 (1973).

[21] C.J. McKinstrie, R. Bingham, Phys. Fluids B 1, 230 (1989).

[22] E.J. Parkes, J. Phys. A: Math. Gen. 21, 2533 (1988).

[23] E. Mjolhus, J. Plasma Phys. 19, 437 (1978). 